\title{
Randomized study of nutritional status and treatment toxicities of oral arginine, glutamine, and Omega-3 fatty acids during concurrent chemoradiotherapy for head and neck cancer patients
}

\author{
Imjai Chitapanarux $^{1}$, Veeradej Pisprasert ${ }^{2}$, Ekkasit Tharavichitkul ${ }^{1}$, Somvilai \\ Jakrabhandu ${ }^{1}$, Pitchayaponne Klunklin ${ }^{1}$, Wimrak Onchan ${ }^{1}$, Bongkot \\ Supawongwattana ${ }^{1}$, Patrinee Traisathit ${ }^{3}$, Sornvichate Rattanachaiwong ${ }^{2}$, and Witiya M \\ Sattasiri $^{4}$ \\ ${ }^{1}$ Division of Therapeutic Radiology and Oncology, Faculty of Medicine, Chiang Mai \\ University, Thailand; ${ }^{2}$ Division of Nutrition, Faculty of Medicine, Khon Kaen University, \\ Thailand; ${ }^{3}$ Department of Statistics, Faculty of Science, Chiang Mai University, Thailand; \\ ${ }^{4}$ Cancer Center, Maury Regional Medical Center, Tennessee, USA
}

Corresponding author: Imjai Chitapanarux, MD, Division of Therapeutic Radiology and Oncology, Faculty of Medicine, Chiang Mai University, 110 Intawarorose Rd, Chiang Mai, 50200, Thailand

Submission Date: Submission Date: November 25, 2015, Acceptance Date: March 2, 2015, Publication Date: March 5, 2015

\begin{abstract}
Background: Patients with head and neck cancer (HNC) undergoing concurrent chemoradiotherapy (CCRT) are at high risk for dysphagia, malnutrition, and immunosuppression. Accordingly, arginine, glutamine, and Omega-3 fatty acidsare immuneenhanced nutrition can promote cellular immunity. We aimed to examine the impact of immunonutrition diet on nutritional status, in addition to CCRT toxicities, within this group of patients.
\end{abstract}

Methods: Forty patients with HNC who were treated with curative CCRT were randomized into group $A(n=20)$, patients who received a regular diet and dietary counseling by a protocol dietician, and group B $(n=20)$, patients who received a regular diet plus immuneenhanced nutrition supplements and dietary counseling by the same protocol dietician. Outcome measures were weight loss, protein and energy intake, serum pre-albumin and albumin, and toxicities of CCRT were evaluated at baseline, weekly and at the end of treatment.

Results: Both groups were well balanced at baseline. One patient from group A $(1 / 20)$ withdrew consent. Seven patients from group B (7/20) withdrew from the study; 1 patient could not tolerate the side effect of chemotherapy and 6 patients could not tolerate the taste of oral immune-enhanced nutrition. A significant loss in total body weight was observed in group A patients $(\mathrm{p}<0.001)$, whereas in group $B$ there no significant weight loss $(\mathrm{p}=0.109)$. Median percentage change from baseline of energy intake was $19.6 \%$, and $22.9 \%$ at the end of treatment for group A and B respectively. The circulating levels of nutritional markers, 
pre-albumin and albumin, decreased after CCRT in both groups. There was a significantly decreased level of albumin in group A compared to that of group B, at the end of treatment. During CCRT; 4 patients (20\%) in group A and 1 patient (5\%) in group B developed grade 3 mucositis, respectively. One patient $(5 \%)$ in group A had grade 3 radiation dermatitis. Grade 3-4 hematologic toxicities, mainly in absolute neutrophil count (ANC), were significantly higher in group A than group B: $20 \%$ versus $0 \%(\mathrm{p}=0.035)$. Over the 7-week period of CCRT, both the intention to treat analysis and per protocol analysis revealed similar results in scaled for all endpoints.

Conclusions: Nutritional counseling and immuno-nutrition can reduce the deterioration of nutrition status and also significantly reduced hematologic and non-hematologic toxicity of CCRT in head and neck cancer.

Keywords: immune-enhanced nutrition, concurrent chemoradiotherapy, head and neck cancer

\section{BACKGROUND:}

Concurrent chemoradiotherapy (CCRT) is one of the standard treatments for locally advanced head and neck cancer. The common toxicities of this treatment include the following: radiation induced oral mucositis, dysphagia, xerostomia, and nausea/vomiting [15] resulted in decreasing of oral intake of the patients. Malnutrition is frequently seen with patients suffering from head neck cancer at diagnosis, which is also aggravated by CCRT. Hematotoxicities are also a form of common toxicity in CCRT. All of these complications leads to unplanned treatment break, prolongation hospitalization, and increased overall treatment time, which has been associated with the poor treatment outcome in these patients [6-10]. A recent narrative review of nutritional interventions in head and neck cancer patients undergoing CCRT recommended to use nutritional counseling and oral nutritional supplements to increase oral intake, and to prevent treatment associated weight loss and treatment break [11]. There are some reports on immune enhanced nutrition using in preoperative or perioperative and postoperative in head and neck cancer and gastrointestinal cancer [12-18]. However, the studies in the application of immune-modulated nutrition for head and neck cancer patients who received CCRT are limited. The primary objective of this study was to measure nutritional parameters (body weight, energy intake, protein intake and nutritional markers). The secondary objective was to evaluate the acute toxicities and compliance of CCRT.

\section{METHODS:}

This non-blinded prospective, randomized study was approved by the institutional ethics committee. Patients were recruited between December 2013 and February 2015. Each patient provided written informed consent before entering in the study. The eligibility criteria were head and neck cancer patients with age more than 18 years intended for CCRT either definitive treatment or adjuvant setting, Eastern Cooperative Oncology Group (ECOG) performance status 0-1 [19]. Exclusion criteria included patients with metastatic disease, history of diabetes mellitus, renal disease, and liver disease. Forty patients were randomly assigned in a 1:1 ratio by a computer program to the control arm (group A); diet counseling 
by protocol dietician only or the study arm (group B); diet counseling by protocol dietician and immunonutrition oral supplementation.

\section{ASSESSMENT:}

Baseline assessment 3-7 days prior to the study was performed for an objective measurement of malnutrition. Blood samples for complete blood cell count (CBC), albumin, and prealbumin were obtained as a marker of nutritional status. Energy and protein intake were calculated at baseline and weekly by a protocol dietician. Body weight and side effect of CCRT were recorded weekly. Acute toxicities of CCRT were evaluated according to the Common Terminology Criteria for Adverse Events (CTCAE), version 4.03 [20] at baseline, and weekly until the end of treatment.

Nutritional counseling and immunonutrition supplements: All patients received individualized nutritional counseling by a protocol dietician on the first week of CCRT and weekly for the whole course of treatment. For group B; patients received immunonutritional supplement [Neo-Mune; Thai Otsuka Pharmaceutical Company, Bangkok, Thailand]. The nutritional formulas used in this study are shown in Table 1. The nutritional supplements were provided for the patients in the study arm by the protocol nurse with 2 glasses per day (1 glass $=250 \mathrm{ml}: 250 \mathrm{kCal}$ ), 1 hour before and after radiotherapy session. Sachets of nutritional supplements were provided to the patients on the weekend and holiday.

Table 1: Nutritional Formulas

\begin{tabular}{|l|c|}
\hline Energy $(\mathrm{kcal} / \mathrm{ml})$ & 1.0 \\
\hline Fat $(\mathrm{g} / \mathrm{L})$ & 28.5 \\
Fat Source & $29 \%$ \\
\hline Corn oil & $52 \%$ \\
Medium chain triglyceride (MCT) & $19 \%$ \\
Fish oil & 61.5 \\
\hline Protein (g/L) & \\
Protein source & $70 \%$ \\
\hline Casein & $20 \%$ \\
Arginine & $10 \%$ \\
Glutamine & \\
\hline
\end{tabular}

Concurrent chemoradiotherapy (CCRT): Definitive curative radiotherapy was administered to the enrolled patients with standard fractionation $2.0 \mathrm{~Gy} /$ fraction for conventional radiotherapy technique to a total of $70 \mathrm{~Gy}$ in 35 fraction; or $2.12 \mathrm{~Gy} /$ fraction for simultaneous integrated boost (SIB) used in nasopharyngeal cancer to a total of $69.96 \mathrm{~Gy}$ in 33 fraction for intensity modulated radiotherapy (IMRT). Adjuvant postoperative radiotherapy was given with standard fractionation $2.0 \mathrm{~Gy} /$ fraction to a total of 60-66 Gy in 30-33 fractions, either by conventional or conformal technique. Concurrent chemotherapy was weekly cisplatin $40 \mathrm{mg} / \mathrm{m}^{2}$ or weekly carboplatin AUC2 (if the creatinine clearance of patient less than 50).

\section{STATISTICAL ANALYSIS:}

From the results of a pilot study, we assume that the weight loss is normally distributed with mean of $5 \mathrm{~kg}$ and a variance of $4 \mathrm{~kg}$. This study is being conducted to examine the effect of 
oral nutrition supplements on weight loss. It is hypothesized that the mean weight loss of group B (study arm) is less than the mean weight loss of group A (control arm). A minimum sample size of 40 patients ( 20 patients per arm) was then calculated to detect a difference in weight loss of $1.9 \mathrm{~kg}$ with a $5 \%$ level of significance (1-sided test) and a power of $90 \%$.

All randomized patients are included in the final intent to treat analysis. Although randomization was the first step to balance known and unknown covariates between study arms, we compared the distribution of all baseline characteristics among arms (chi-square test for qualitative variables and Wilcoxon test for continuous variables). Median of nutritional parameters during treatment between control arm and study arm were analyzed using the Wilcoxon-Mann Whitney test. Data related to incidence, prevalence, or frequency (symptoms, cancer sites, and nutritional status categories) were expressed as number and/or percentage, with age, energy and protein intakes being expressed as the median, interquartile range (IQR). Continuous variables were analyzed using Wilcoxon-Mann Whitney test, and categorical variables were evaluated by the chi-square test.

\section{RESULTS:}

A total of 40 patients were recruited in the study, including 26 men (65\%) and 14 women (35\%), with a median age of 51.5 years (IQR 48.0-60.0). The patient and clinical characteristics are shown in Table 2. Most of the patients were well-balanced between two groups. Laboratory assessments (complete blood count, renal function, serum electrolytes, liver function test, and thyroid hormone) at baseline in both groups were comparable. A comparison with the control arm for the main study's end points with the intention to treat analysis was performed, although this study was completed only 32 patients. The number of patients remaining in the study decreased mainly in group B by approximately $20 \%$ over 7 weeks of treatment. One patient in group A withdrew consent, whereas 7 patients $(35 \%)$ in group B withdrew from the study (due to intolerable of the taste of immune-enhanced nutrition in 6 patients, and due to the toxicity of CCRT in 1 patient). Per protocol analysis was also studied in these 32 patients. The consort diagram was shown in Figure 1.

Table 2: Baseline Characteristics of the patients

\begin{tabular}{|c|c|c|c|}
\hline IQR & Group A & Group B & p-value \\
\hline Median Age(years)(IQR) & $54.0(49.5-60.5)$ & $49.5(41.5-55.0)$ & $0.064 *$ \\
\hline Sex & & & \\
\hline Male & $13(65 \%)$ & $13(65 \%)$ & $1.000 \dagger$ \\
\hline Female & $7(35 \%)$ & $7(35 \%)$ & \\
\hline Type of Cancer & & & \\
\hline Nasopharynx & $9(45 \%)$ & $8(40 \%)$ & $0.599 \dagger$ \\
\hline Oral cavity & $3(15 \%)$ & $5(25 \%)$ & \\
\hline Oropharynx & $6(30 \%)$ & $4(20 \%)$ & \\
\hline Hypopharynx & $2(10 \%)$ & $3(15 \%)$ & \\
\hline \multicolumn{4}{|l|}{ Stage } \\
\hline III & $6 \quad(30 \%)$ & $2 \quad(10 \%)$ & $0.286 \dagger$ \\
\hline IVA & $10 \quad(50 \%)$ & $13 \quad(65 \%)$ & \\
\hline IVB & $4 \quad(20 \%)$ & $5 \quad(25 \%)$ & \\
\hline $\begin{array}{l}\text { Karnofsky performance } \\
\text { status score }\end{array}$ & & & $0.490 \dagger$ \\
\hline
\end{tabular}




\begin{tabular}{|l|l|l|l|}
\multicolumn{1}{|c|}{1} & \multicolumn{1}{|c|}{$\begin{array}{c}15(75 \%) \\
5(25 \%)\end{array}$} & \multicolumn{1}{c|}{$7(65 \%)$} & \\
\hline $\begin{array}{l}\text { Median Baseline body } \\
\text { weight (kg)(IQR) }\end{array}$ & $56.3(50.0-59.5)$ & $60.0(50.3-67.0)$ & $0.267^{*}$ \\
\hline $\begin{array}{l}\text { Median Baseline serum pre- } \\
\text { albumin (mg/dL)(IQR) }\end{array}$ & $0.208(0.129-0.256)$ & $0.252(0.137-0.273)$ & $0.189^{*}$ \\
\hline $\begin{array}{l}\text { Median Baseline serum } \\
\text { albumin (mg/dL)(IQR) }\end{array}$ & $4.4(4.1-4.5)$ & $4.4(4.1-4.6)$ & $0.806^{*}$ \\
\hline
\end{tabular}

*Wilcoxon-Mann Whitney test

$\dagger$ Chi-square test

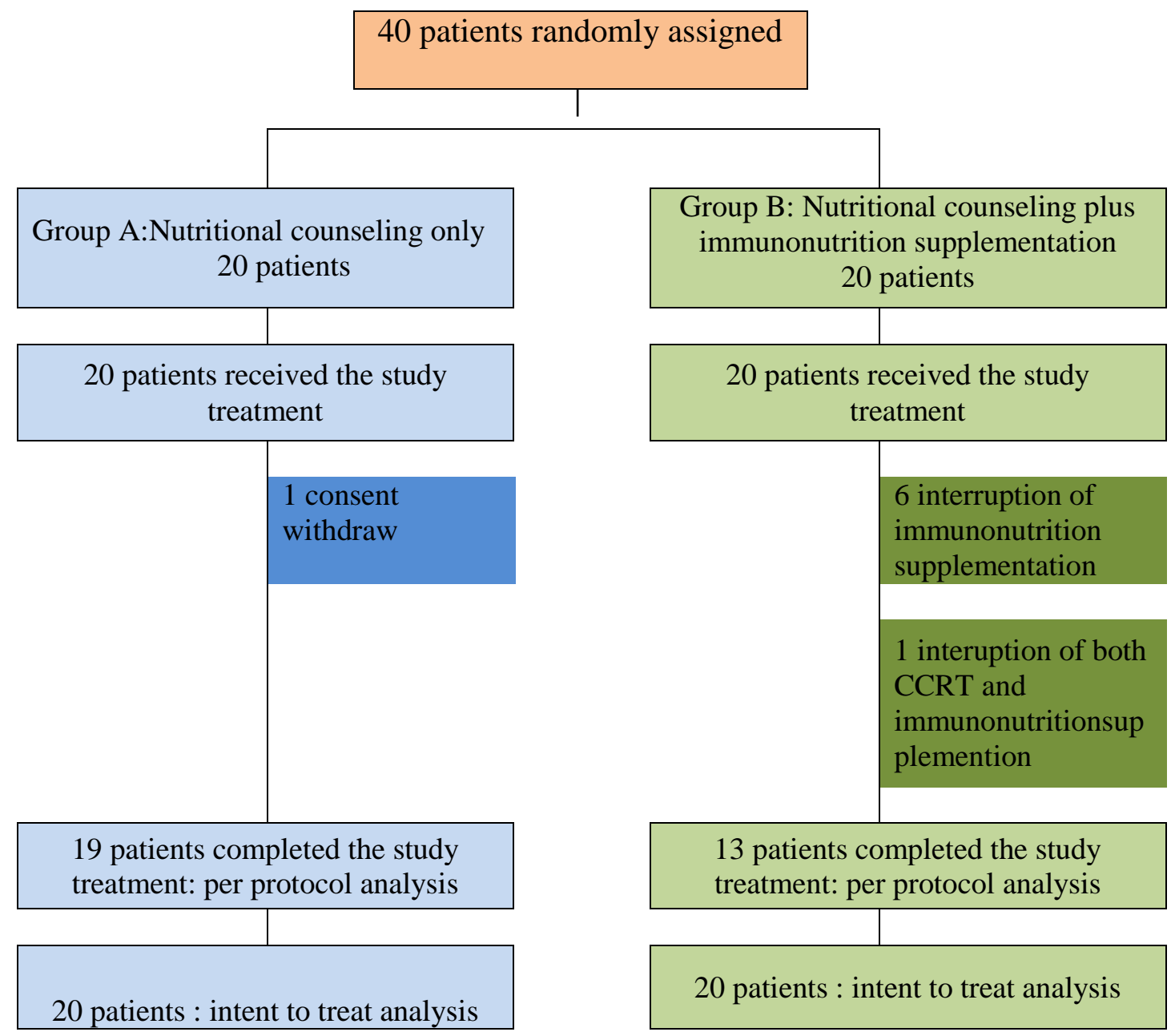

Figure 1: Consort diagram

\section{Nutritional parameter:}

\subsection{Weight loss}

The median weight changed significantly from $56.3 \mathrm{~kg}$ (IQR 50.0-59.5) at baseline to $47.0 \mathrm{~kg}(\mathrm{IQR} 45.5-50.5)$ at the end of treatment $(\mathrm{p}<0.001)$ in group A, but did not significantly decrease from 60.0 (IQR 50.3-67.0) at baseline to 53.0 (45.0-62.0) at the end of treatment $(\mathrm{p}=0.109)$ in group $\mathrm{B}$. (Table 3). In the per-protocol group changes were similar in scale. 
Table 3: Median body weight

\begin{tabular}{|c|rrr|rrr|c|}
\hline \multirow{2}{*}{ Variables } & \multicolumn{3}{|c|}{ Group A $(\mathbf{n = 2 0})$} & \multicolumn{3}{c|}{ Group B $(\mathbf{n = 2 0})$} & p-value* \\
\cline { 2 - 8 } & $\mathbf{n}$ & \multicolumn{1}{c|}{ Median } & $(\mathbf{I Q R})$ & $\mathbf{n}$ & Median & (IQR) & \\
\hline Weight** & & & & & & & \\
Baseline & 20 & 56.3 & $(50.0-59.5)$ & 20 & 60.0 & $(50.3-67.0)$ & 0.267 \\
Week 3 & 20 & 52.5 & $(49.0-56.5)$ & 15 & 59.1 & $(49.0-67.0)$ & 0.151 \\
$\begin{array}{l}\text { End of } \\
\text { treatment }\end{array}$ & 13 & 47.0 & $(45.5-50.5)$ & 6 & 53.0 & $(45.0-62.0)$ & 0.251 \\
\hline
\end{tabular}

Note.n, Number of patients in each group; $N$, Total number of patients

* Wilcoxon-Mann Whitney test for median comparison

$* *$ Median body weight at the end of treatment of overall $(p<0.001)$ and group A

$(p<0.001)$ decreased from baseline but maintained in group $B(p=0.109)$

1.2 Energy Intake

Median percentage change of energy intake decreased overtime in both groups (Figure 2). However, the median energy intake of group B was significantly higher than group A. The Figure 3 shows the median energy intake in this study. In the per-protocol group, changes were similar in scale.

\section{Median \% change energy intakes}

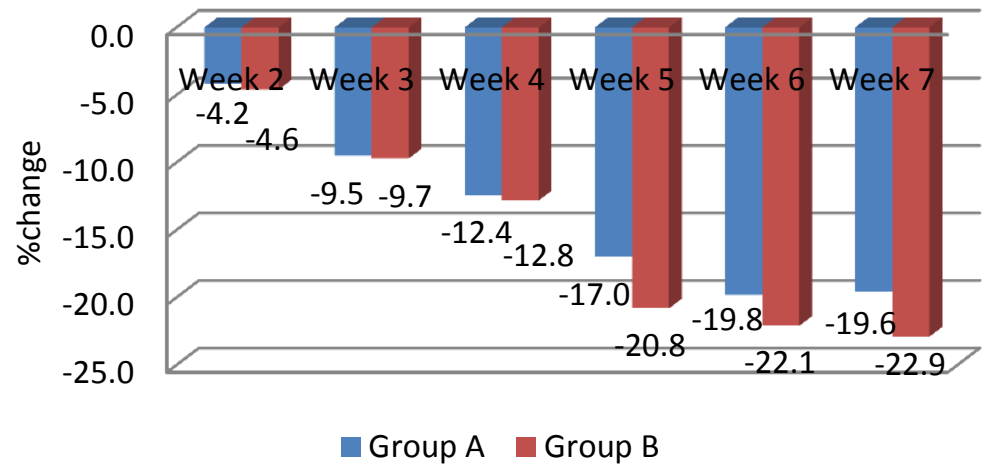

Figure 2: Median percentage change energy intake

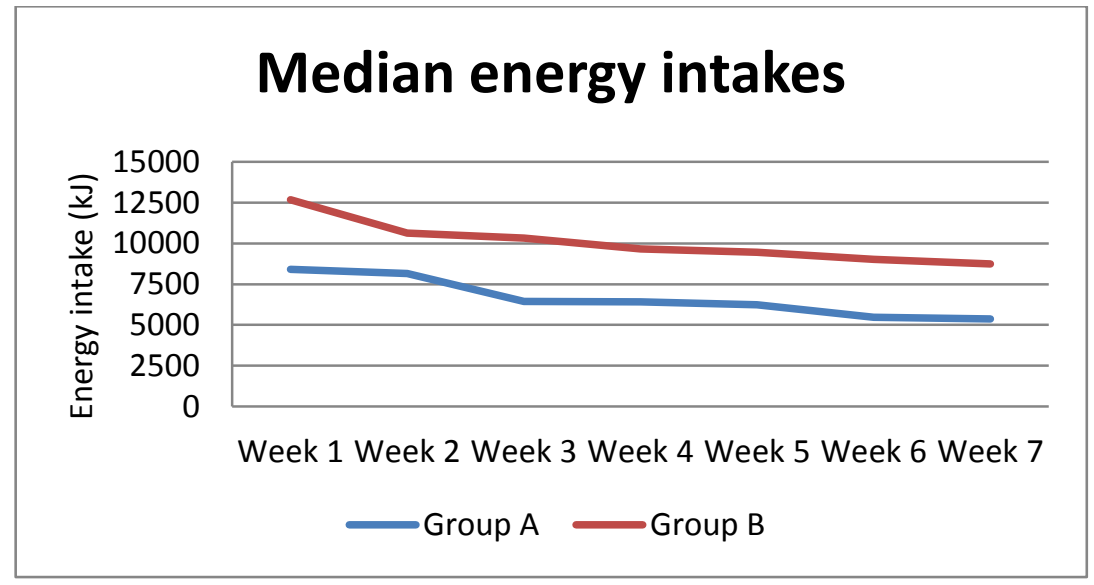

Figure 3: Median energy intakes 


\subsection{Protein Intake}

Median percentage change of protein intake decreased over time during CCR Tin both groups of patient (Figure 4). However, patients who received immune- enhanced nutrition had increase protein intake compared to those who did not during the second week (Figure 5). In the per-protocol group, we found the statistically significant higher protein intake in group B in the $2^{\text {nd }}, 4^{\text {th }}$, and $5^{\text {th }}$ week of treatment.

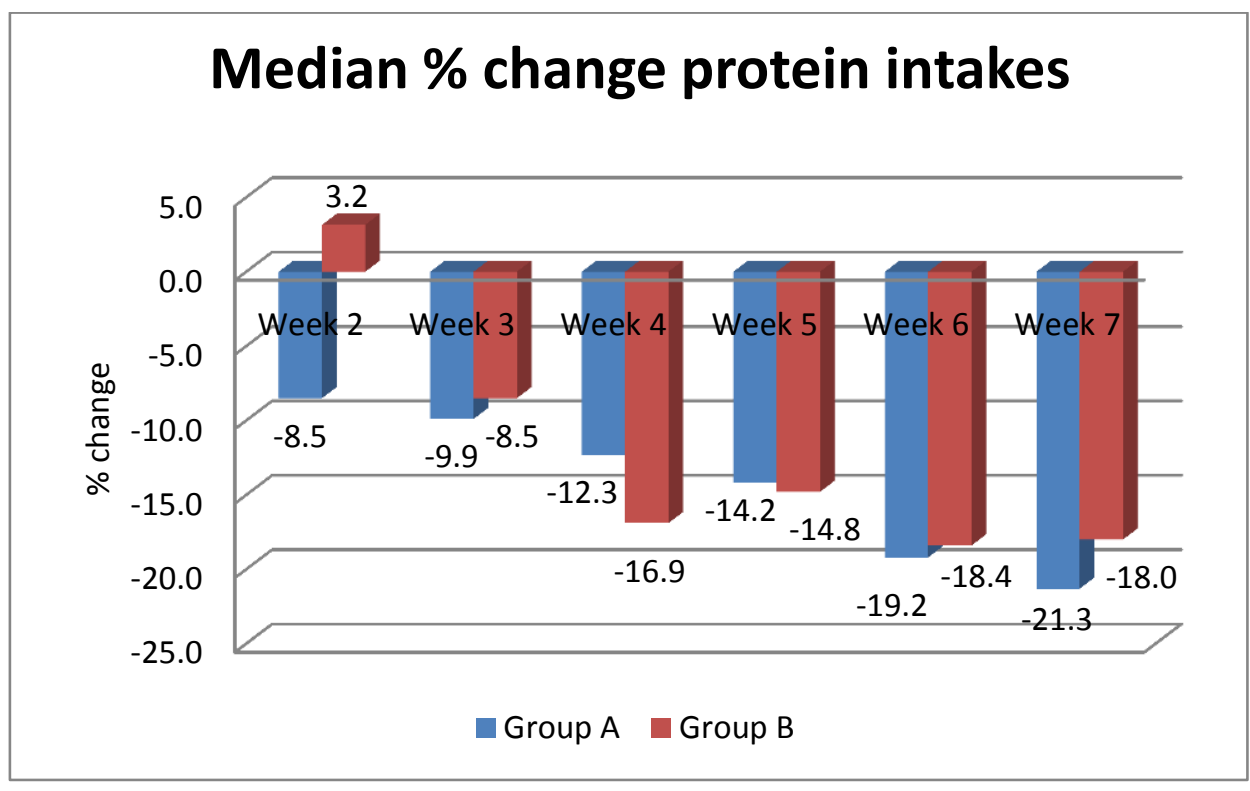

Figure 4: Median percentage change protein intakes

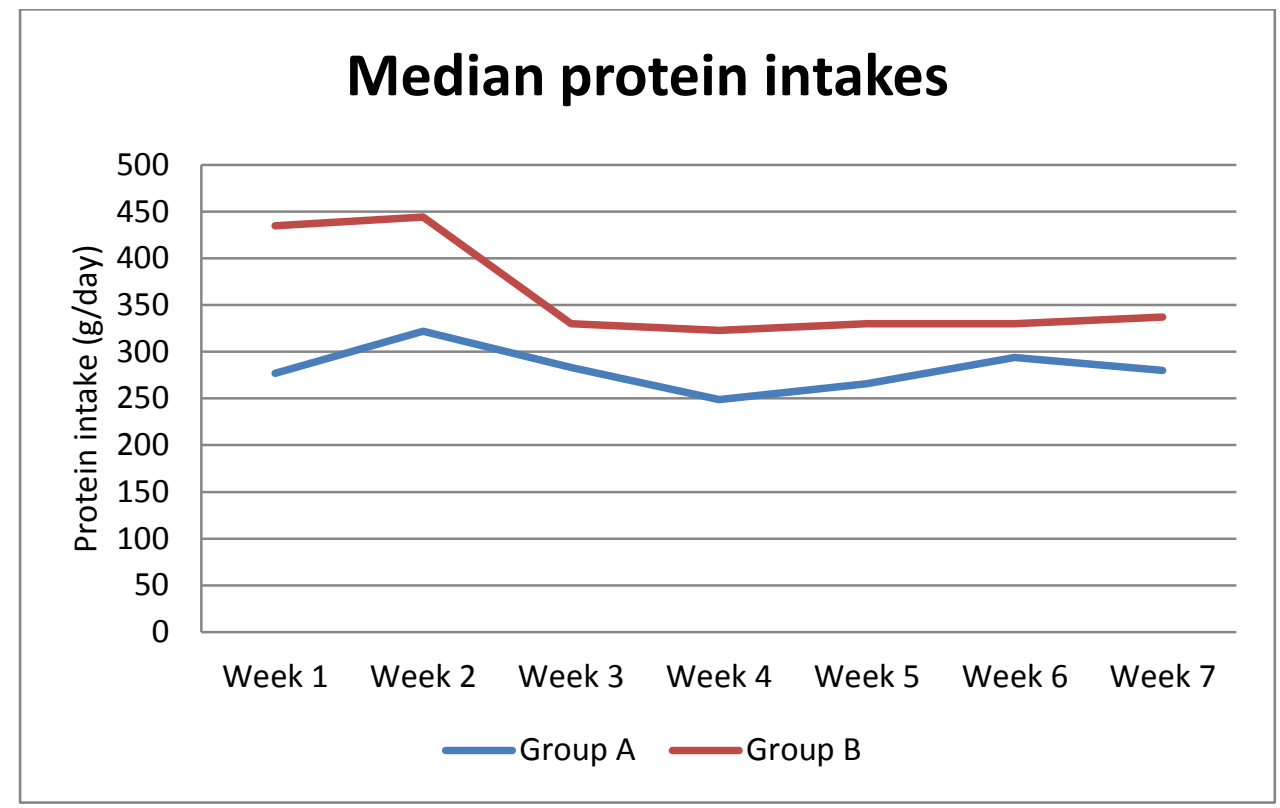

Figure 5: Median protein intakes

\subsection{Nutritional markers}

Both nutritional markers (albumin and pre-albumin) decreased during CCRT in both groups of patient. However, median circulating albumin at the end of treatment of group B was significantly higher than group A $(p=0.028)$, whereas median pre-albumin did not significantly different between the groups $(\mathrm{p}=0.272)$ (Table 4$)$. We found similar results in the per-protocol group. 
Table 4. Median circulating levels of nutritional markers

\begin{tabular}{|c|c|c|c|c|c|c|c|c|}
\hline \multirow{2}{*}{ Variables } & \multicolumn{3}{|c|}{ Baseline } & \multirow{2}{*}{$\begin{array}{c}\text { p- } \\
\text { value* }\end{array}$} & \multicolumn{3}{|c|}{ End of treatment } & \multirow{2}{*}{$\begin{array}{c}\text { p- } \\
\text { value* }\end{array}$} \\
\hline & $\mathbf{n}$ & Median & (IQR) & & $\mathbf{n}$ & Median & (IQR) & \\
\hline \multicolumn{9}{|l|}{ Pre-albumin } \\
\hline Group A & 20 & 0.188 & $(0.136-0.260)$ & \multirow[t]{2}{*}{0.502} & 17 & 0.154 & $(0.087-0.199)$ & \multirow[t]{2}{*}{0.812} \\
\hline Group B & 17 & 0.205 & $(0.164-0.269)$ & & 14 & 0.138 & $(0.115-0.185)$ & \\
\hline \multicolumn{9}{|l|}{ Albumin } \\
\hline Group A & 20 & 4.4 & $(4.1-4.5)$ & 0.806 & 19 & 3.9 & $(3.7-4.2)$ & 0.028 \\
\hline Group B & 20 & 4.4 & $(4.1-4.6)$ & & 14 & 4.3 & $(4.0-4.6)$ & \\
\hline
\end{tabular}

Note. $n$, Number of patients in each group; $N$, Total number of patients *Wilcoxon-Mann Whitney test for median comparison

\section{Toxicities of CCRT:}

For non-hematologic toxicity, radiation induced oral mucositis was the most common affliction for both groups of patients. 20\% (4/20) of group A developed grade 3 mucositis, whereas only $5 \%(1 / 20)$ of patients did in group B. Radiation dermatitis was found to be $5 \%(1 / 20)$ and $0 \%$ in group A and B respectively. For hematologic toxicity, the severe grade 3-4 hematologic toxicities were also found to be significantly higher in group A than group $B$ significantly $(p=0.035)$. Whereas we did not find any grade 3-4 hematologic toxicities in group B patients, but we found grade 3, and 4 neutrophil count decrease in 3 patients and 1 patient from group A respectively. One patient in group A had grade 3 thrombocytopenia. These were the causes of extension of the overall treatment time (OTT) between the two groups, 55 days in group A versus 51 days in group B. However, this did not make a significant statistical difference, measured at $\mathrm{p}=0.316$. These severe toxicities were also the cause of the incomplete planning of CCRT. The patients in group B had statistically significantly higher complete CCRT than patients from group A, $\mathrm{p}=0.013$. We found similar results in the per-protocol group, in higher grade 3-4 hematologic toxicities in group A patients, but not statistically significant $(p=0.132)$ as intent to treat analysis.

\section{Adverse event of Immune-enhanced nutrition:}

No immune-enhanced nutrition related serious adverse events occurred. The most common observed events were nausea (35\%), due to the taste of the samples. Additionally, nausea was also the cause of intolerance and withdrew from the study before the last week of CCRT.

\section{DISCUSSION:}

A recent review from Bossola $M$ [11] suggested that nutritional counseling and oral nutritional supplements should be used to increase dietary intake and to prevent treatmentassociated weight loss and interruption of radiotherapy or concurrent chemoradiotherapy for head and neck cancer patients. However, all the evidence [21-28] used drew from standard enteral nutrition, rather than an immune modulating enteral nutrition formula. We are aware of only a few published randomized trials that studied immune-enhanced nutrition in CCRT for head and neck cancer [29-30].

The present study shows that, head and neck cancer patients undergoing CCRT who received nutritional counseling or had immune-enhanced nutrition supplementation both had 
weight loss overtime during the treatment. A study by Arnold et al. [21] also found the same results as this present study, with that weight loss can occur in both groups (either received nutritional supplements or not) during the observation period of 6 months. Median weight loss was about $5.2 \%$ within patients from group A and $4.2 \%$ in group B. Study of Vasson MP et al. [30] had 5.6\%, and 6.2\% weight loss at the end of treatment in patients who received standard nutrition and immune-enhanced nutrition, respectively. The patient who received immunonutrition in our study had less percentage weight loss than the Vasson MP et al [30] study, as nearly $50 \%$ of patients in their study had esophageal cancer, whereas none of our patients did. The median energy and protein intake in both groups of patients were also decreased significantly from the first week of treatment until the end of CCRT. Our results demonstrate that adding immune-enhanced nutrition may improve energy and protein levels in certain patients.

This study demonstrated that immune-enhanced nutrition can significantly slow the deterioration of serum albumin in patients treated by CCRT. However, pre-albumin level may be more specific in detecting a malnourished state than albumin level. Our results did not demonstrate that immune-enhanced nutrition had a significant effect on pre-albumin levels.

Severe grade 3-4 hematological toxicities were strongly affected as much as $20 \%$ by CCRT especially in patients who did not receive immuno-nutrition, whereas the patients who received immuno-nutrition had none. Sunpaweravong S. et al. [31] reported 5.7\% of patients receiving immuno-nutrition developed severe neutropenia, which was higher than ours. However, their study only focused on esophageal cancer patients who were treated with different chemotherapy regimens from ours by using cisplatin $75 \mathrm{mg} / \mathrm{m}^{2} /$ day and the field of radiotherapy for esophageal cancer is wider than head and neck cancer. Moreover, a higher proportion of group A patients compared to those of group B developed high grade oral mucositis and dermatitis during CCRT. As a result, it appears that immune-nutrition during CCRT could also have a positive effect on non-hematologic toxicities occurrence. This would be the useful benefit to decrease the numbers of patients who need the CCRT treatment break. Although it has no statistically significant difference in the median overall treatment time, the patients who received immuno-nutrition can complete their CCRT treatment in a shorter period of time. We also performed per protocol analysis and found that the baseline characteristics in both groups were almost similar in every variable, except for the statistically significant younger age in group B patients (48 VS 54 years old; $p=0.003$ ). However, all the endpoints, including weight, energy and protein intake, nutritional marker, toxicities of CCRT, overall treatment time, and rate of incomplete planned of chemotherapy were similar in scale.

A limitation in our study is our sample size. This study is being conducted to examine the effect of oral nutrition supplements on weight loss. A minimum sample size of 40 patients (20 patients per arm) was calculated to detect a difference in weight loss of $1.9 \mathrm{~kg}$ with a $5 \%$ level of significance (1-sided test) and a power of $90 \%$. However, the number of patients including in the analysis is lower than the statistical number of 30 in each group which is scientifically desirable.

\section{CONCLUSION:}

In conclusion, the present study shows that immune-enhanced nutrition combined with nutritional counseling in head and neck cancer patients undergoing CCRT is beneficial in reducing the deterioration of nutritional status and also the severity of CCRT toxicities. 
List of abbreviations used: HNC: head and neck cancer; CCRT: concurrent chemoradiotherapy; ANC: absolute neutrophil count; ECOG: Eastern Cooperative Oncology Group; CBC: complete blood cell count; CTC AE: Common Terminology Criteria for Adverse Events; SIB: simultaneous integrated boost; IMRT: intensity modulated radiotherapy; AUC: area under curve; IQR: inter-quartile range

Competing interest: The authors declare that they have no competing interests.

Authors' Contributions: IC conceived and coordinated the study, analyzed the data, and drafted the manuscript, VP coordinated and analyzed the study, ET, SJ, PK, WO, BS participated in acquisition of data. PT performed the statistical analysis. WMS and SR helped to draft the manuscript. All authors read and approved the final manuscript.

Acknowledgements and Funding: The immune enhanced nutrition in this study was supported by Thai Otsuka Pharmaceutical Company, Bangkok, Thailand.

\section{REFERENCES:}

1. Bonner JA, Harari PM, Giralt J, Azarnia N, Shin DM, Cohen RB, Jones CU, Sur R, Raben D, Jassem J, Ove R, Kies MS, Baselga J, Youssoufian H, Amella N, Rowinski EK, Ang KK: Radiotherapy plus cetuximab for squamous-cell carcinoma of the head and neck. N Engl J Med 2006; 354:567-578.

2. Brizel DM, Albers ME, Fisher SR, Scher RL, Richtsmeier WJ, Hars V, George SL, Huang AT, Prosnitz LR: Hyperfractionated irradiation with or without concurrent chemotherapy for locally advanced head and neck cancer. $N$ Engl J Med. 1998;18:1798-1804.

3. Colasanto JM, Prasad P, Nash MA, Decker RH, Wilson LD: Nutritional support of patients undergoing radiation therapy for head and neck cancer. Oncology. 2005;19:371-379.

4. Ng K, Leung SF, Johnson PJ, Woo J: Nutritional consequences of radiotherapy in nasopharynx cancer patients. Nutr Cancer. 2004;49:156-161.

5. Sliver HJ, Dietrich MS, Murphy BA: Changes in body mass, energy balance physical function, and inflammatory state in patients with locally advanced head and neck cancer treated with concurrent chemoradiation after low-dose induction chemotherapy. Head Neck. 2007;29:893-900.

6. Bernier J, Domenge C, Ozsahin M, Matuszewska K, Lefebvre JL, Greiner RH, Giralt J, Maingon P, Rolland F, Bolla M, Cognetti F, Bourhis J, Kirkpatrick A, van Glabbeke M, European Organization for Research and Treatment of Cancer Trial 22931: Postoperative irradiation with or without concomitant chemotherapy for locally advanced head and neck cancer. N Engl J Med. 2004;350:1945-1952.

7. Bieri S, Bentzen SM, Huguenin P, Allal AS, Cozzi L, Landmann C, Monney M, Bernier J: Early morbidity after radiotherapy with or without chemotherapy in advanced head and neck cancer. Experience from four nonrandomized studies. Strahlenther Onkol. 2003;179:390-395.

8. Cooper JS, Pajak TF, Forastiere AA, Jacobs J, Campbell BH, Saxman SB, Kish JA, Kim HE, Cmelak AJ, Rotman M, Lustig R, Ensley JF, Thorstad W, Schultz CJ, Yom SS, Ang KK: Long-term follow-up of the RTOG 9501/intergroup phase III trial, 
postoperative concurrent radiation therapy and chemotherapy in high-risk squamous cell carcinoma of the head and neck. Int J Radiat Oncol Biol Phys. 2012; 84:11981205.

9. Lin A, Jabbari S, Worden FP, Bradford CR, Chepeha DB, Teknos TN, Liao JJ, Nyquist GG, Tsien C, Schipper MJ, Urba S, Wolf GT, Eisbruch A: Metabolic abnormalities associated with weight loss during chemoirradiation of head-and-neck cancer. Int J Radiat Oncol Biol Phys. 2005;63:1413-1418.

10. Nquyen NP, Moltz CC, Frank C, Vos P, Smith HJ, Bhamidipati PV, Karlsson U, Nguyen PD, Alfieri A, Nguyen LM, Lemanski C, Chan W, Rose S, Sallah S: Aspiration rate following rate following chemoradiation for head and neck cancer: an underreported occurrence. Radiotherapy and Oncology. 2006; 80: 302-306.

11. Bossola M: Nutritional intervention in head and neck cancer patients undergoing chemoradiotherapy: a narrative review. Nutrients. 2015; 7:265-276.

12. De Luis DA, Izaola O, Cuellar L, Terroba MD, Arranz M, Fernandez N, Aller R: Effect of c-reactive protein and interleukins blood levels in postsurgery arginineenhance enteral nutrition in head and neck cancer patients. EurJ Clin Nutr. 2003:57:96-99.

13. De Luis DA, Izaola O, Cuellar L, Terroba MC, Aller R: Randomized clinical trial with an enteral arginine-enhanced formula in early postsurgical head and neck cancer patients. Eur J Clin Nutr. 2005; 58:1505-1508.

14. De Luis DA, Arranz M, Aller R, Izaola O, Cuellar L, Terroba MC: Immunoenhanced enteral nutrition, effect on inflammatory markers in head and neck cancer patients. Eur J Clin Nutr. 2005;59:145-147.

15. De Luis DA, Izaola O, Cuellar L, Terroba MC, Martin T, Aller R: Clinical and biochemical outcomes after a randomized trial with a high dose of enteral arginine formula in postsurgical head and neck cancer patients. Eur J Clin Nutr. 2007;61:200204.

16. Riso S, Aluffi P, Brugnani M, Farinetti F, Pia F, D’Andrea F: Postoperative enteral immunonutrition in head and neck cancer patients. Clin Nutr. 2000;19:407-412.

17. Reynolds JV, Daly JM, Zhang S, Evantash E, Shou J, Sigal R, Ziegler MM: Immunomodulatory mechanisms of arginine. Surgery. 1988; 104:142-151.

18. van Bokhorst-De Van Der Schueren MA, Quak JJ, von Blomberg-van der Flier BM, Kuik DJ, Langendoen SI, Snow GB, Green CJ, van Leeuwen PA: Effect of perioperative nutrition, with and without arginine supplementation, on nutritional status, immune function, postoperative morbidity, and survival in severely malnourished head and neck cancer patients. Am J Clin Nutr. 2001; 73:323-332.

19. Oken MM, Creech RH, Tormey DC, Horton J, Davis TE, McFadden ET, Carbone PP: Toxicity and Response Criteria of the Eastern Cooperative Oncology Group. Am J Clin Oncol.1982; 5:649-655.

20. Common Terminology Criteria for Adverse Events (CTCAE) version 4.03. U.S. Department of Health and Human Seminar, National Institute of Health, National Cancer Institute:www.eortc.be.

21. Arnold C, Richter MP: The effect of oral nutritional supplements on head and neck cancer. Int J Radiat Oncol Biol Phys. 1989;16:1595 - 1599. 
22. Nayel H, el-Ghoneimy E, el-Haddad S: Impact of nutritional supplementation on treatment delay and morbidity in patients with head and neck tumors treated with irradiation. Nutrition. 1992;8: 13- 18.

23. Gonçalves Dias MC, de Fátima Nunes Marucci M, Nadalin W, Waitzberg DL: Nutritional intervention improves the caloric and proteic ingestion of head and neck cancer patients under radiotherapy. Nutr Hosp. 2005;20:320 - 325.

24. Ravasco P, Monteiro-Grillo I, Marques Vidal P, Camilo ME: Impact of nutrition on outcome, a prospective randomized controlled trial in patients with head and neck cancer undergoing radiotherapy. Head Neck. 2005; 27:659 - 668.

25. Isenring EA, Bauer JD, Capra S: Nutrition support using the American Dietetic Association medical nutrition therapy protocol for radiation oncology patients improves dietary intake compared with standard practice. J Am Diet Assoc. 2007;107:404 - 412.

26. Paccagnella A, Morello M, da Mosto MC, Baruffi C, Marcon ML, Gava, Baggio V, Lamon S, Babare R, Rosti G, Giometto M, Boscolo-Rizzo P, Kiwanuka E, Tessarin M, Caregaro L, Machiori C: Early nutritional intervention improves treatment tolerance and outcomes in head and neck cancer patients undergoing concurrent chemoradiotherapy. Support Care Cancer. 2010;18:837-845.

27. van den Berg MG, Rasmussen-Conrad EL, Wei KH, Lintz-Luidens H, Kaanders JH, Merkx MA: Comparison of the effect of individual dietary counselling and of standard nutritional care on weight loss in patients with head and neck cancer undergoing radiotherapy. Br J Nutr. 2010;104:872 -877.

28. Valentini V, Marazzi F, Bossola M, Miccichè F, Nardone L, Balducci M, Dinapoli N, Bonomo P, Autorino R, Silipigni S, Giuliani F, Tamanti C, Mele MC, Martorana GE: Nutritional counselling and oral nutritional supplements in head and neck cancer patients undergoing chemoradiotherapy. J Hum Nutr Diet. 2012;25:201-208.

29. Machon C, Thezenas S, Dupuy AM, Assenat E, Michel F, Mas E, Senesse P, Cristol JP: Immunonutrition before and during radiochemotherapy: improvement of inflammatory parameters in head and neck cancer patients. Support Care Cancer. 2012;20:3129-3135.

30. Vasson MP, Talvas J, Perche O, Dillies AF, Bachmann P, Pezet D, Achim AC, Pommier P, Racadot S, Weber A, Ramdani M, Kwiatkowski F, Bouteloup C: Immunonutrition improves functional capacities in head and neck and esophageal cancer patients undergoing radiochemotherapy: a randomized clinical trial. Clin Nutr.2014; 33:204-210.

31. Sunpaweravong S, Puttawibul P, Ruangsin S, Laohawiriyakamol S, Sunpaweravong P, Sangthawan D, Pradutkanchana J, Geater A: Randomized study of antiinflammatory and immune-modulatory effects of enteral immunonutrition during concurrent chemoradiotherapy for esophageal cancer. Nutr Cancer.2014; 66(1):1-5. 